\title{
A New Control Structure for Multi-Terminal dc Grids to Damp Inter-Area Oscillations
}

\section{Eriksson, Robert}

Published in:

IEEE Transactions on Power Delivery

Link to article, DOI:

10.1109/TPWRD.2014.2364738

Publication date:

2014

Link back to DTU Orbit

Citation (APA):

Eriksson, R. (2014). A New Control Structure for Multi-Terminal dc Grids to Damp Inter-Area Oscillations. IEEE Transactions on Power Delivery, 31(3), 990-998. https://doi.org/10.1109/TPWRD.2014.2364738

\section{General rights}

Copyright and moral rights for the publications made accessible in the public portal are retained by the authors and/or other copyright owners and it is a condition of accessing publications that users recognise and abide by the legal requirements associated with these rights.

- Users may download and print one copy of any publication from the public portal for the purpose of private study or research.

- You may not further distribute the material or use it for any profit-making activity or commercial gain

- You may freely distribute the URL identifying the publication in the public portal

If you believe that this document breaches copyright please contact us providing details, and we will remove access to the work immediately and investigate your claim. 


\title{
A New Control Structure for Multi-Terminal dc Grids to Damp Inter-Area Oscillations
}

\author{
Robert Eriksson, Member, IEEE
}

\begin{abstract}
This article analyzes the control structure of the multi-terminal dc (MTDC) system to damp ac system interarea oscillations through active power modulation. A new control structure is presented that maximizes the relative controllability without the need for communication among the de terminals.

In point-to-point high voltage de (HVDC) transmission, the active power modulation of the two terminals occurs in opposite directions. In this case the control direction is given and only needs to be phase compensated to align for maximal damping. In the case of MTDC systems the control direction interrelates with the active power modulation share of the de terminals and the relative controllability depends on this.

The new control structure eliminates the need of communication between the dc terminals by performing dc voltage feedback loop shaping. This makes it possible to modulate the power in one terminal and let the other terminals react on the dc voltage change. Through loop shaping, where the feedback gain varies in the frequency plane as compared to the regular droop design, the control direction is aligned with the direction of highest relative controllability. The loop shaping takes place without influencing the high frequency or steady-state gain.

Simulations in the Nordic32 test system show the validity of the proposed controller and its structure.
\end{abstract}

Index Terms-Coordinated control, de voltage control, high voltage direct current HVDC, multi-terminal dc MTDC, Nordic32, small signal stability.

\section{INTRODUCTION}

$\mathbf{T}$ HE increase of renewable energy sources in the European power system, together with development of the liberalized market have changed the operation of the power system. As a response to this change, the power technologies used in the transmission system need to become more flexible, observable and controllable [1]. Multi-terminal dc (MTDC) grids would move the power system toward this, if designed as overlaid meshed grid, and has the potential to connect renewable energy sources such as offshore wind farms. Future dc grids are likely to be based on voltage source terminals (VSC) which have some advantages over the current source terminal, used in classical high voltage dc (HVDC). However, there are many challenges to be solved before MTDC grids are realized in practice.

In multi-terminal configurations, the dc voltage plays a crucial role when it comes to system control. Therefore, it is of interest to spread the dc voltage control amongst different terminals which can be obtained by using a so-called voltage droop control [2]-[5]. The main advantage of such a distributed voltage controller is that all controlling terminals react upon a change in the dc voltage, similarly to the way

R. Eriksson is with the Center for Electric Power and Energy, Technical University of Denmark, 2800 Kgs. Lyngby, Denmark. Email: reriks[at]elektro.dtu.dk. a synchronous generator reacts on frequency changes by the primary control. The small time scale makes the control of the dc voltage more challenging, especially when one takes into account the fact that the dc voltage at the different buses varies as a result of the power flows through the lines. Much research has been carried out on the dc voltage droop control itself; the effects of the controller gains on the ac system and ancillary services have not received too much attention so far.

Power oscillation damping (POD) control has been studied for many decades and the supplementary control design for point-to-point HVDC has been well described in literature [6]-[12]. Having several controllable devices, e.g. MTDC, in the system raises the need of coordination. The European Electric Grid Initiative (EEGI) has listed the increase of coordination and a pan-European reliability assessment as essential parts of the future "smarter" grid [13]. Coordination between controllable devices exists to only a limited extent today, especially between devices located in different zones and controlled by different operators. As more devices are installed, coordinated strategies must be incorporated into the operation and controller design [14]. If the controllable devices are not coordinated, negative interactions may occur among the steady-state and electro-mechanical damping controllers [14]. Ref. [15]-[17], have shown that coordinated control of HVDC links can improve the dynamic stability of the ac system and increase the secure transfer capacity.

The supplementary control structure for POD can be designed either as centralized or decentralized. Both reactive and active power can be considered, reactive power modulation follows straightforward control design as it is modulated individually in the converters. The proposed control structure can easily be combined with reactive power control following standard design procedures. In centralized control communication of the set-point change is needed from the controller to the terminals which obviously is a drawback e.g. reduced reliability and data latency [18]. In decentralized control the controller is placed in one or several of the terminals and the other terminals change their power according to the dc voltage droop. The drawback in this case is instead that the controllability is not fully used. This is since the power change relies on the dc voltage droop and the power change might not be in the direction of highest damping (highest relative controllability) for the specific inter-area mode [19].

This paper contributes with a new decentralized control structure for POD based on dc voltage loop-shaping to maximize the relative controllability. In addition, it presents a comparison of present control structures for inter-area oscillation damping. This new decentralized control structure provides the maximal relative controllability and does not imply commu- 
nication between terminals, instead it takes advantage of $\mathrm{dc}$ voltage loop shaping control.

The paper continues as follows: Section II describes the linear modeling of the MTDC-VSC grid and ac system and, develops the combined model. Section III performs linear analysis and presents the theory of the phase compensating controller. Next section presents the new control structure and compares it to present control structures. In Section $\mathrm{V}$ the maximum relative controllability is found and the novel dc voltage closed loop shaping is explained. Section VI presents the simulation result in the Nordic32 test system including the five terminal dc grid in PowerFactory/Digsilent.

\section{LINEAR MODELING OF DC AND AC SYSTEMS}

To study interactions, models of the dc and ac systems are developed, described in the two following subsections.

\section{A. The dc system}

Modeling of the VSC is in the decoupled $d-q$ reference frame where the direct, $d$-axis, is aligned with the positive voltage sequence at the point of common coupling and the quadrature, $q$-axis, is displaced by 90 degrees. The phaselocked loop (PLL) tracks changes in the system frequency and adjusts the speed of rotation of the reference frame accordingly. The PLL is implemented in PowerFactory with the standard PLL using $K_{P-P L L}=3$ and $K_{I-P L L}=1$ which gives sufficiently fast response. Further details are left out of the scope of this article, readers are referred to [20] for a detailed description. In this article the power/current is defined as positive flowing from the ac grid to the dc grid.

The inner current control loop is the most important control of the VSC and relates $d-q$ quantities to the ac system quantities. In this study the dynamic of the inner current loop can be neglected since it has much higher bandwidth than the dc voltage control. This outer control loop gives the reference values of the $d$ and $q$ currents i.e., $I_{d-r e f}$ and $I_{q-r e f}$. Thus, the $I_{q}$ and $I_{d}$ currents are related to the reactive and active power injections, respectively. Furthermore, the $I_{d}$ is directly coupled to the dc voltage. The active power output $P_{d c}$ of terminal $k$ is given by

$$
P_{d c-k}=-\left(P_{a c-k}+P_{l o s s-k}\right)=-V_{d c-k} I_{d-k}
$$

where $P_{\text {loss }-k}$ the power loss and $V_{d c-k}$ the dc voltage at converter $k$, respectively.

Operation of the terminals is important for the dynamic behavior of the dc system. Each terminal can either work in $P$-control, $V_{d c}$-control mode or a combination of those modes. The combined mode is called droop (proportional) control implying the terminals share the burden to maintain the dc voltage i.e. power balance. For a stable system, at least one terminal needs to support the dc voltage.

In droop mode, the power offset $P_{d c-k-e}$ in terminal $k$ due to voltage deviation is given by

$$
P_{d c-k-e}=K_{p}\left(V_{d c-k}-V_{d c-r e f-k}\right)
$$

where $K_{p}$ is the constant voltage droop gain and $V_{d c-r e f-k}$ is the reference value of the dc voltage. A value of $K_{p}=\infty$

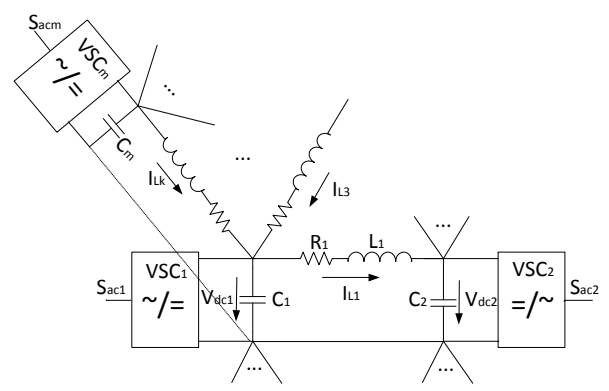

Figure 1. A generalized MTDC grid.

indicates the terminal is in strict voltage control and $K_{p}=0$ means the terminal is in power control mode.

Modeling of the dc grid is given by the capacitor charging and inductor current basic differential equations. The generalized MTDC grid is depicted in Fig. 1.

The differential equation describing the voltage change at node $k$ is given by

$$
\dot{V}_{d c-k}=\frac{1}{C_{c a p-k}}\left(I_{d-k}-\sum_{j \in \Omega_{k}} I_{L-j}\right) \quad \forall k
$$

$\Omega_{k}$ contains the indexes of all branches connected to node $k$ with line currents of branch $j$ being $I_{L-j}$ and, $C_{c a p-k}$ the capacitance of converter $k$.

The current change in line $j$ connecting nodes $k$ and $m$ is described by

$$
\dot{I}_{L-j}=\frac{1}{L_{L-j}}\left(-R_{L-j} I_{L-j}+V_{d c-k}-V_{d c-m}\right) \quad \forall j
$$

where $R$ is the resistance and, $L$ the inductance and, $V_{d c-k}$ and $V_{d c-m}$ are the dc voltages at node $k$ and $m$, respectively.

The open loop dc system has the structure, based on (3) and (4), as follows

$$
\dot{x}_{d c}=A_{d c} x_{d c}+B_{d c} u_{d c}
$$

where $x_{d c}=\left[V_{d c-1} \ldots V_{d c-m} I_{L-1} \ldots I_{L-p}\right]$ for $m$ nodes and $p$ lines $x_{d c} \in \mathbb{R}^{m+p}$ and the control variables $u_{d c}=\left[\begin{array}{lll}I_{1} & \ldots I_{m}\end{array}\right]$, $u_{d c} \in \mathbb{R}^{m}$. The closed loop system using only droop control has the feedback signal as follows

$$
u_{d c}=-K_{p} C_{d c} x_{d c}
$$

with $C_{d c}$ having one on the main diagonal and zero elsewhere, $C_{d c} \in \mathbb{R}^{m \times(m+p)}$. To design the local feedback controller for the dc voltage several aspects need to be considered and there is trade off among steady-state dc voltage error and disturbance attenuation over the frequency spectrum. The closed loop response has earlier been carefully analyzed in [5], where the droop gain impact on the dc system frequency response is analyzed using singular value decomposition (SVD). From the MIMO model of the MTDC system the droop gain is found through the SVD by varying the droop gain, selecting the largest gain with some margin, fulfilling the dc voltage steady-state error for example $10 \%$ off-set. 


\section{B. The ac system}

The injected power $S_{a c-k}$ to the ac grid, neglecting the losses, by VSC $k$ is given by

$$
S_{a c-k}=V_{d-k}\left(I_{d-k}-j I_{q-k}\right) .
$$

The ac power system can be described by a set of nonlinear algebraic and differential equations. To study inter-area oscillations a linearized model is sufficient which as described by

$$
\begin{aligned}
& \dot{x}_{a c}=A_{a c} x_{a c}+B_{a c} I_{d c} \\
& y_{a c}=C_{a c} x_{a c}
\end{aligned}
$$

where $x_{a c}=\left[\delta \omega E_{q}^{\prime}\right], \delta$ and $\omega$ are the generator rotor angles and speeds, respectively, $E_{q}^{\prime}$ is the internal generator voltage and, $y_{a c}$ is the output vector. $B_{a c} I_{d c}$ relates the ac state variables and power injections of the MTDC system.

\section{C. ac and dc system}

Combining the linear ac and dc models gives the equations as follow

$$
\begin{aligned}
\dot{x} & =\left[\begin{array}{c}
\dot{x}_{a c} \\
\dot{x}_{d c}
\end{array}\right]= \\
& =\left[\begin{array}{cc}
A_{a c} & B_{a c} \\
0 & A_{d c}-B_{d c} K_{p} C_{d c}
\end{array}\right]\left[\begin{array}{l}
x_{a c} \\
x_{d c}
\end{array}\right]+\left[\begin{array}{ll}
0 & B_{d c}
\end{array}\right]\left[\begin{array}{c}
0 \\
u_{d c}
\end{array}\right] \\
& =A x+B u \\
y & =C x
\end{aligned}
$$

In the linearized model, the dc system is independent of the ac system. However, the ac system depends on the dc system, in particular, the power injections of the terminals.

To show the difference of the ac and dc systems the relation of the quantities are given in Table I. The different time scales becomes obvious studying the system inertia and energy [21].

Table I

QUANTITY RELATION IN DC AND AC SYSTEMS

\begin{tabular}{lcc} 
Characteristic & $\mathrm{ac}$ & $\mathrm{dc}$ \\
\hline Power balance & $\mathrm{f}$ & $V_{d c}$ \\
Voltage change & $V \sin (\delta)$ & $\Delta V_{d c}$ \\
Steady-state connection impedance & $\mathrm{X}$ & $\mathrm{R}$ \\
Real power transfer & $\frac{V_{j} V_{k} \sin (\delta)}{X_{j k}}$ & $\frac{V_{d c} \Delta V_{d c}}{R_{d c}}$ \\
System inertia & $\mathrm{J}$ & $C_{c a p}$ \\
System energy & $\frac{J \omega^{2}}{2}$ & $\frac{C V_{d c}^{2}}{2}$
\end{tabular}

\section{LINEAR ANALYSIS}

The system description (10) and (11) is analyzed by the eigenvalue and eigenvector analysis as

$$
\begin{aligned}
A \varphi_{R_{i}} & =\lambda_{i} \varphi_{R_{i}} \\
\varphi_{L i}^{T} A & =\varphi_{L i}^{T} \lambda_{i}
\end{aligned}
$$

where $\varphi_{R i}$ and $\varphi_{L i}$ are the right and left eigenvectors for eigenvalue $i, \lambda_{i}$. Since the objective is to damp inter-area oscillations in the ac system, one needs to find the mode of interest for further analysis of this mode.

In the analysis the control variables, control directions, and feedback signals (measurements) are of main importance [22]. The set of control variables are already defined by the terminals and their placement defines the influence on the ac system. The control direction is not pre-defined as the active power can be modulated independently in the terminals with the restriction of keeping the power balance (dc voltage) giving the freedom of dimension $m-1$.

The relative controllability of different input directions for a particular eigenvalue $i$ can be expressed as

$$
b_{i}=\left(\varphi_{L i}^{T} B\right)^{T} .
$$

Furthermore, the relative observability of different outputs can be expressed as

$$
o_{i}=C \varphi_{R i} .
$$

The feedback signals, which are some linear combination of the states, are the measurements.

The residue, which is the product of the relative controllability and observability, can be expressed by

$$
R_{i}=o_{i} b_{i}^{T}
$$

There are other methods to analyse the system which are in detailed described and evaluated in [23]. For instance the Popov-Belevitch-Hautus is based on singular values of the controllability and observability matrices for a certain mode. The geometric measures, instead, provides similar measure as the residue with the advantage of being normalized. This is the quantitative measure of shifting eigenvalue $i$ in the complex plane by the feedback loop $\alpha H_{a c}(s)$. Larger value of $R_{i}$ means lower feedback gain $\alpha$, i.e. control effort, is needed to shift the eigenvalue. It is desirable to use lower control effort and therefore larger value of the residue is beneficial. To shift the eigenvalue to the left in the complex plane the phase of the feedback loop, for the eigenvalue of interest, needs to be phase compensated to 180 degrees (or 0 degrees depending on if one uses positive or negative feedback). Then the eigenvalue can be shifted to the left by the gain $\alpha$. To create such feedback loop the lead-lag control block can be used combined with a washout filter which commonly is the high-pass filter to avoid steady state deviation. The controller $H_{a c}(s)$ is given by

$$
H_{a c}(s)=\frac{s T_{w}}{1+s T_{w}}\left(\frac{1+s T_{2}}{1+s T_{1}}\right)^{\gamma},
$$

and the control signal is then given by

$$
U_{d c}(s)=\alpha H_{a c}(s) B_{a c}(s) C_{a c-k} X_{a c}(s) .
$$

To design the supplementary controller for an inter-area mode the control direction and feedback signal need to be defined.

The relative controllability is determined by the physical location of the VSC and can therefore not be affected by the POD controller, however, it can be maximal utilized by an optimized control structure. Moreover, the relative controllability is usually higher when the VSC is connected electrically 
close. As for all controllers, centralized or decentralized, the maximal relative controllability of a mode cannot be changed, as the system topology then would need to be changed.

The next section describes different control structures and their impact.

\section{SUPPLEMENTARY CONTROL DESIGN}

The generator bus frequency or voltage deviation are local measurements with often high observability of inter-area oscillations. Measures, like generator angle deviation of internal voltage $E^{\prime}$, would require communication to the controller, and for some control structures, additional communication from the controller to the different converters. In [24], a criterion to select the most suitable input-output pair for the design of PSS for WPP is presented. This considers the control design limitations with respect to local feedback signal selections. Further analysis may be carried out to find the most suitable feedback signal when modeling the system with input and output disturbance signals but is left out of this paper. However, the control structure of MTDC systems for supplementary inter-area oscillations damping can be constructed in several ways. An overview of the different control structures is given in Table IV-A. In this section different options are stated and the new control structure design is presented.

The control signals can be provided to the terminals in two ways as follows

- centralized control which sends the modulating set-point to each terminal,

- decentralized control where one or several local controllers provide the modulating set-point to the local terminal and let the power modulation in the other terminals happen through the dc voltage control.

In each group there are different possibilities described below.

\section{A. Control structure}

The control problem can be divided into two parts which are the observability and controllability, combined, the measure of residue. The focus of this article is to study the relative controllability and its dependency on the control structure. Clearly, linear analysis indicates that the residue highly depends on the control structure where the measurement signals are related to the observability of the system. Different control structures are discussed below

\section{Decentralized control}

a Decentralized basic control structure without communication uses a local measurement and modulates the power in one terminal, shown in Fig. 2(a). Consequently the other terminals react upon the dc voltage change resulting in power change according to the dc voltage droop defining the control direction.

b Several decentralized basic controllers, as described in (a), without communication among them are installed in several or all terminals. The control direction is the sum of the power set-points depending on the dc voltage droop settings, the control structure is shown in Fig. 2(b).
Table II

OVERVIEW OF THE DIFFERENT CONTROL STRUCTURES.

\begin{tabular}{lccccc} 
& $\mathrm{a}$ & $\mathrm{b}$ & $\mathrm{c}$ & $\mathrm{d}$ & $\mathrm{e}$ \\
\hline Centralized & & & $\mathrm{X}$ & $\mathrm{X}$ & $\mathrm{X}$ \\
Decentralized & $\mathrm{X}$ & $\mathrm{X}$ & & & \\
\hline Communication & & & $\mathrm{X}$ & $\mathrm{X}$ & \\
\hline Local meas. & $\mathrm{X}$ & $\mathrm{X}$ & $\mathrm{X}$ & & $\mathrm{X}$ \\
WAMS & & & & $\mathrm{X}$ & $(\mathrm{X})$ \\
\hline $\begin{array}{l}\text { Relative controllab. } \\
\text { Low }\end{array}$ & $\mathrm{X}$ & & & & \\
$\begin{array}{l}\text { Medium } \\
\text { High }\end{array}$ & & $\mathrm{X}$ & & & \\
\hline
\end{tabular}

\section{Centralized control}

c Centralized basic control with terminal communication uses a local measurement and communicates the power-set points to each terminal as depicted in Fig. 2(c).

d Centralized master control with full communication where wide-area measurements signals (WAMS) are sent to the master controller. The controller derives the power set-points and sends out these to all the terminals, this is depicted in Fig. 2(d). This control structure relies on communication from the measurement points to the controller and from the controller to the terminals.

\section{B. New control structure - Decentralized control with dc voltage loop shaping}

e The new control structure maximizes the relative controllability by dc voltage closed loop shaping. Instead of communication, the power change is communicated in the $\mathrm{dc}$ voltage. Only one controller receives the power modulation signal, consequently, the other terminals react on the dc voltage change. This occurs according to the designed closed loop gain, aiming to maximize the relative controllability. The structure is shown in Figs. 2(e) and 3, and details are given in Subsection V.

Since this paper focuses on the small signal stability Hac and Hdc will not vary depending on the disturbance. However, they may change depending on the operating point of the system as in all cases based on linearization.

\section{Maximizing the Relative CONTROLlabiLity of THE AC/DC SYSTEM}

In the new method the control signals are instead transferred via the dc voltage where the novel idea is to optimize the relative controllability by introducing a new dc voltage control. Changing the reference signal in one terminal, $u_{r e f-d c-m}(s)$, changes the output of the other terminals according to the closed loop gain evaluated at the certain frequency of the mode. The dynamic of the dc voltage is very fast, thus, the delay in power change response in the other terminals is negligible valid in the frequency band of the inter-area oscillations. The new dc voltage control method performs closed loop shaping in the frequency band of inter-area oscillations, which makes the terminals to take different share, defining the control directions. 


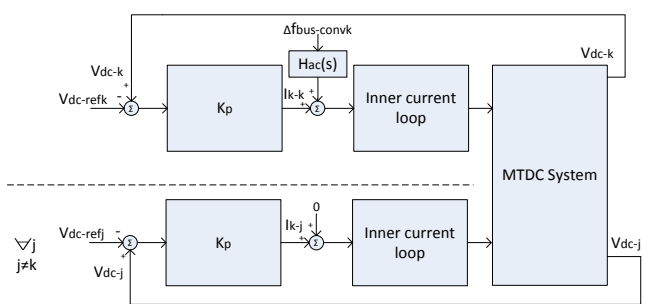

(a) Basic feedback

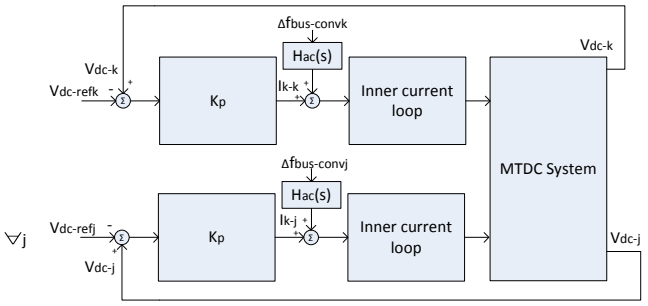

(b) Decentralized control

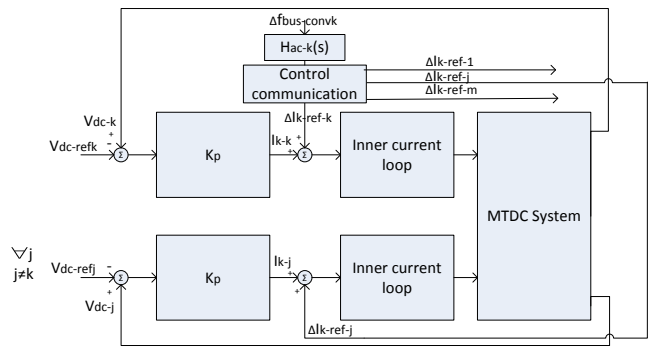

(c) Basic feedback and communication to the terminals

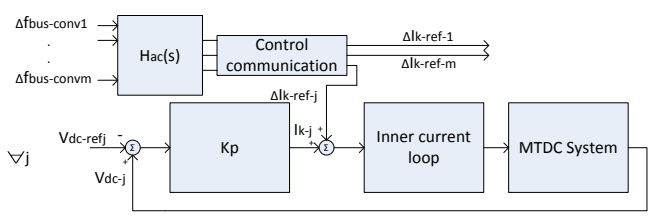

(d) WAMS signals and master controller with communication to the terminals

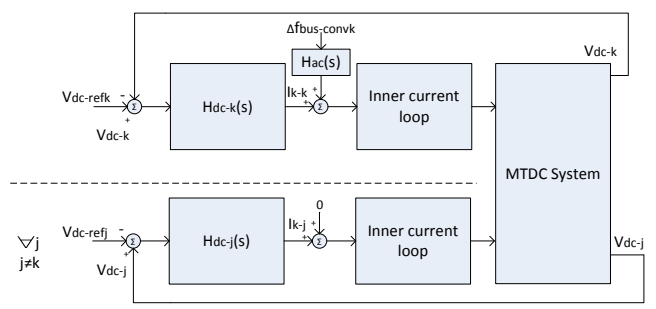

(e) dc voltage loop shaping design

Figure 2. Five different control structures for POD control.

The power change in all the terminals is described by the closed loop transfer function of the MTDC system given by

$$
\begin{aligned}
Y_{d c}(s) & =\frac{G_{d c}(s)}{1+K_{p} G_{d c}(s)} u_{r e f-d c}(s)= \\
& =B_{M T D C}(s) u_{r e f-d c}(s) .
\end{aligned}
$$

Using constant dc voltage droop, changing the set-point in one of the terminals changes the power in the other terminals in the opposite direction, i.e. phase shifted 180 degrees. This is since the injected power balance is disturbed and the terminals
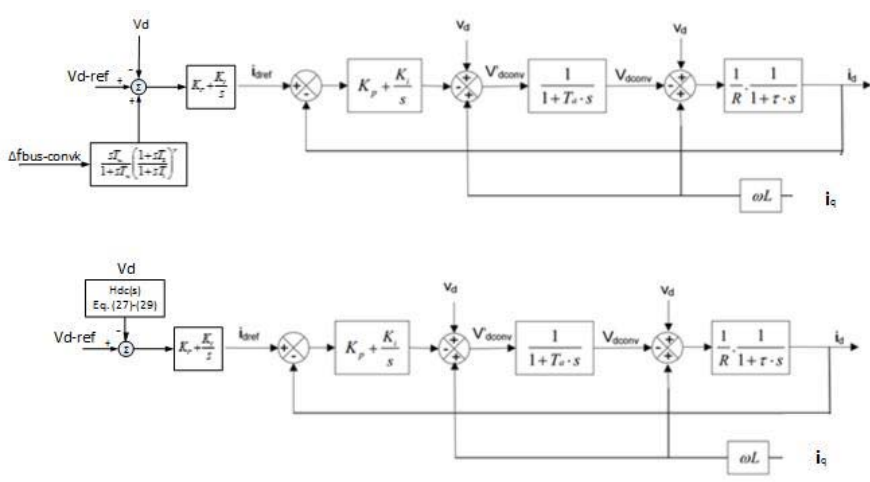

Figure 3. Detailed block diagram of the proposed control structure.

participating in the droop control reacts by changing their power to maintain the dc voltage. It can be realized that the droop control does not necessarily provide the best relative controllability as the share among the terminals, i.e. control direction, may not be aligned with the direction of highest relative controllability. Therefore a new method is proposed which performs loop shaping of the closed loop de voltage mainly influenced at the frequency of the inter-area mode. The loop shaping aims to maximize the relative controllability without the use of communication among the terminals. The power converter to be selected to install the main damper controller is evaluated from the residue analysis, the location with largest absolute value of the residue should be selected unless there are other reasons to place it somewhere else.

\section{A. Optimal control direction}

The residue for eigenvalue $i$ and feedback signal $k$, which is a linear combination of the states, is given by

$$
\begin{aligned}
R_{i}(k, k) & =C_{a c-k} V_{R-i} V_{L-i} B_{d c} B_{M T D C}\left(j \omega_{i}\right)= \\
& =R_{i}^{\prime} B_{M T D C}\left(j \omega_{i}\right) .
\end{aligned}
$$

where $B_{M T D C}\left(j \omega_{i}\right)$ is the closed loop transfer function of the MTDC system evaluated at the frequency of mode $i$.

The size of the residue indicates the combined relative observability and controllability, thus larger value indicates proper location for the damping controller.

To maximize the relative controllability, the control direction should be aligned with a certain row in the residue $R_{i}^{\prime}$ in (20). However, this is subjected to certain conditions which may interfere with this. To find a unique solution we let the control signals be in the range between \pm 1 and the sum of the control signals be equal to zero for power balance.

This can be formulated, for each feedback signal $k$, as an optimization problem to find the direction of highest gain for eigenvalue $i$ as follows

$$
\begin{array}{ll}
\max & \left|R_{i-k}^{\prime} u_{i-m}\right|=\left|\sum_{\substack{\forall j \\
j \neq k}} R_{i-k}^{\prime}(k, j) u(j)\right| \\
\text { s.t. } & \left|u_{i-m}(k)\right|=1 \\
& \sum_{j} u_{i-m}(j)=0 \\
& \left|u_{i-m}(j)\right| \leq 1 \quad \forall j
\end{array}
$$


where $u_{k-i}$ is the control direction of Converter $k$ for eigenvalue $i$.

This is an optimization problem with complex numbers, thus, the problem possesses a continuous phase as in contrast to real number optimization problems. The linear programming cannot be used to solve this problem although it seems linear. Nevertheless, the problem can be transformed to second order cone programming (SOCP) by

$$
\sum\left|\begin{array}{l}
\mathfrak{R}\left(R_{i}\right) \\
\mathfrak{I}\left(R_{i}\right)
\end{array}\right|_{2}
$$

where $\mathbb{R}(\times)$ and $\mathfrak{I}(\times)$ denote the real and imaginary parts, respectively. This second order cone programming problem can be solved using e.g. GAMS.

Solving (21)-(24) gives the optimal control direction $u_{m-i}^{*}$ which maximizes the residue of eigenvalue $i$ for feedback signal $k$.

The optimization is very fast and can be run if the operating point is changed to be re-tune the parameters. Similar to other controllers where a change in the operating point of the ac system changes the true model. Although, dc power flow changes has minor influence on the control direction, modulating the power in one converter, this can easily be considered by retuning of the controller applying the optimization procedure.

\section{B. dc voltage loop shaping}

The MTDC system should respond in the direction of highest gain, as previously described, implying the need for re-shaping of the closed loop dc voltage response. This should appear without affecting the steady-state and high frequency gain implying different responds over frequency occurring through all-pass filters. The new dc voltage control has the following structure

$$
K_{\text {opt }}(s)=K_{p}\left[\begin{array}{ccc}
H_{d c-1}(s) & 0 & \ldots \\
0 & H_{d c-2}(s) & 0 \ldots \\
0 & \ldots & \ddots
\end{array}\right]
$$

The local dc voltage controller $m$ where the damping controller is installed is given by

$$
H_{d c-m}(s)=1
$$

For each terminal $j \neq m$, depending on the value of the optimal direction $u_{i-m}^{*}(j)$, the feedback gain is given by

$$
\begin{gathered}
\text { if } u_{i-m}^{*}(j)<0 \\
H_{d c-j}(s)=\frac{1+s T_{b}}{u_{i-m}^{*}(j)+s T_{b}} \frac{u_{i-m}^{*}(j)-s T_{a}}{1-s T_{a}} \\
\text { if } u_{i-m}^{*}(j)>0 \\
H_{d c-j}(s)=\left(\frac{1+s T_{b}}{\sqrt{u_{i-m}^{*}(j)}+s T_{b}}\right)^{2}\left(\frac{\sqrt{u_{i-m}^{*}(j)}-s T_{a}}{1-s T_{a}}\right)^{2} \\
\text { if } u_{i-m}^{*}(j)=0 \\
H_{d c-j}(s)=\frac{1+s T_{b}}{\varepsilon+s T_{b}} \frac{\varepsilon-s T_{a}}{1-s T_{a}}
\end{gathered}
$$

where $\varepsilon$ is a small number and $T_{a}$ and $T_{b}$ are the parameters in the all-pass filter. The parameters $T_{a}$ and $T_{b}$ will be the same for each converter. $T_{a}$ and $T_{b}$ are designed such that the phase shift occurs just before the frequency of the mode of interest and the next phase shift occurs just after. The steepness of the filter is designed sufficient slope in order not to interfere with the disturbance attenuation of any input disturbance, similar to [5]. These filters do not change the high frequency or steadystate gains of each local dc voltage controller.

Thus, the closed loop transfer function is given by

$$
\begin{aligned}
Y_{d c}(s) & =\frac{G_{d c}(s)}{1+K_{o p t}(s) G_{d c}(s)} u_{r e f-d c}(s)= \\
& =B_{M T D C}^{*}(s) u_{r e f-d c}(s)
\end{aligned}
$$

and has the output direction $u_{i-m}^{*}$ for eigenvalue $i$ and the damping controller in terminal $m$ i.e.

$$
u_{i-m}^{*}=B_{M T D C}^{*}\left(j \omega_{i}\right) u_{r e f-d c-m}\left(j \omega_{i}\right)
$$

resulting in the maximum residue value $R_{i-m}^{*}$.

The ac POD design in (17), belongs to a very robust control design with large phase margin and is therefore not sensitive to parameter changes, like change in mode frequency. This design is commonly used in generator excitation system PSS and POD for single HVDC links, working at various operating points. The dc voltage control structure is also robust as it works within a range of frequencies, considered in the design (26). Moreover, the sensitivity of the input direction can be analyzed through the singular value decomposition or looking at the sensitivity of the residues.

\section{FIVE TERMINAL EXAMPLE INTEGRATED IN THE NORDIC TEST SYSTEM}

A five terminal MTDC system is integrated in the Nordic32 test system, as depicted in Fig. 4. The linear analysis of the Nordic system indicates a poorly damped inter-area mode, Mode 1, at $0.57 \mathrm{~Hz}$ [25]. The objective is to increase the damping of this mode by active power modulation in the MTDC system. In this example system the VSC is connected electrically close to one of the generators with high participation in the mode of interest. If this is not the case, instead located electrically far from the participating generators, the relative controllability is significantly reduced. However, the method maximizes the relative controllability for any placement and provides better damping compared to conventional control structure as the input is not necessarily aligned with the direction providing maximal damping in the latter case. The method can design the filter parameters for any system topology and depends on this topology. If the system under study has several modes, which are poorly damped, another controller can be installed in parallel following the same design procedure as earlier described. This could mean that one converter will have two POD controllers connected or that they are placed in different converters.

In this case study only one local frequency measurement is used to show that the mode can be damped quite well using the new method. However, the method works for both for decentralized and centralized control. 
The new dc voltage loop shaping control utilizes the optimal control direction by the local dc voltage controllers. In Tab. III the residues are compared and the absolute value of the residue is most relevant.

The table shows that the better location for the damping controller is Converter 5. It can also be noted that the residue is increased by a factor of slightly more than 3 which implies the new control method is this factor more effective than basic control. The optimal control direction for the controller in Converter 5 is $u_{\text {Mode1-5 }}^{*}=\left[\begin{array}{lllll}1 & 1 & 0 & -1 & -1\end{array}\right]$. Furthermore, simulations are run for two disturbances and the result is compared for the two different control structures.

\section{Table III}

RESIDUES

\begin{tabular}{ccccc} 
Conv & $R_{\text {Mode } 1-\text { Basic }}$ & $\left|R_{\text {Mode } 1-\text { Basic }}\right|$ & $R_{\text {Mode1-opt }}^{*}$ & $\left|R_{\text {Mode1-opt }}^{*}\right|$ \\
\hline 1 & $0.1469+\mathrm{i} 0.0156$ & 0.1477 & $0.3667+\mathrm{i} 0.0290$ & 0.3678 \\
2 & $0.0217-\mathrm{i} 0.0025$ & 0.0219 & $0.1191-\mathrm{i} 0.0075$ & 0.1194 \\
3 & $0.0315+\mathrm{i} 0.0051$ & 0.0319 & $0.4412+\mathrm{i} 0.0292$ & 0.4421 \\
4 & $0.0281+\mathrm{i} 0.0053$ & 0.0286 & $0.3847+\mathrm{i} 0.0314$ & 0.3860 \\
5 & $0.2066+\mathrm{i} 0.0036$ & $\mathbf{0 . 2 0 6 6}$ & $0.6831+\mathrm{i} 0.0321$ & $\mathbf{0 . 6 8 3 9}$
\end{tabular}

\section{A. Case study 1}

The disturbance in this case is a load increase by $10 \%$ at $\mathrm{t}=1 \mathrm{~s}$, corresponding to $200 \mathrm{MW}$, at bus 4072 for the duration of $1 \mathrm{~s}$. The result is shown in Fig. 5, where the angle difference of Generators 4072 and 4063 are displayed which are representative of the system's behavior. In Figs. 6 and 7 the power change is shown of each terminal of the two POD controllers. The peak power is similar in both POD controllers for Converter 5 but differs for the other terminals. The new POD controller, based on dc voltage closed loop shaping, utilizes the other terminals to a larger extent as it takes the control direction of highest damping into account. This new

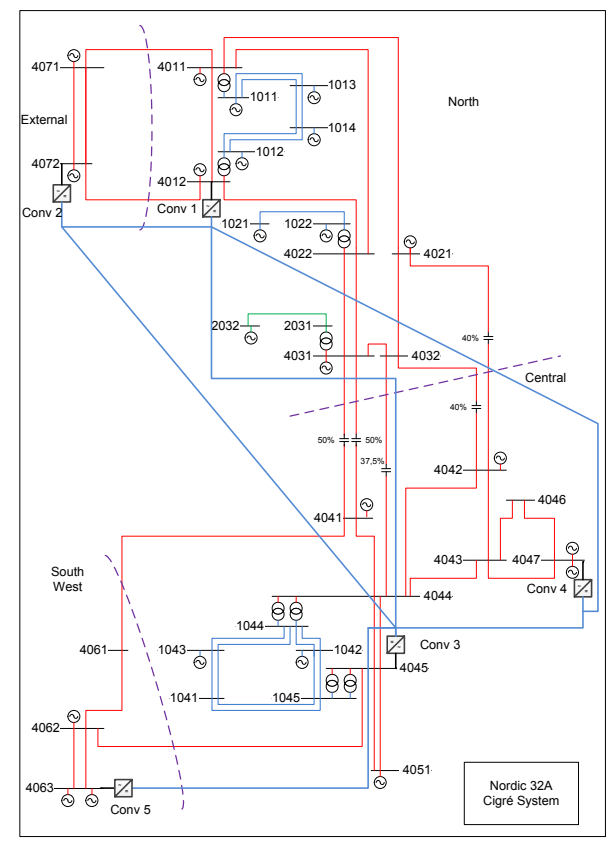

Figure 4. Overview of the Nordic test system with five terminal MTDC system. control structure has a positive effect on the POD compared to the basic control where the proportional dc voltage droop is applied.

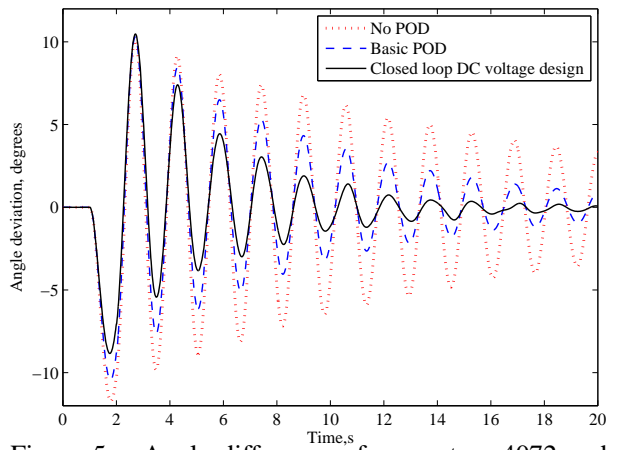

Figure 5. Angle difference of generators 4072 and 4063.

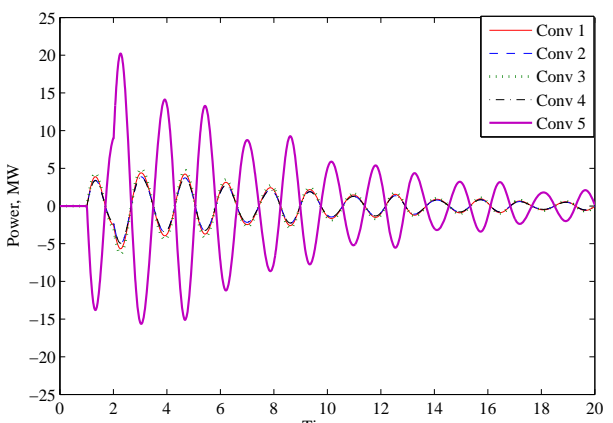

Figure 6. Power modulation of the terminals for the case of POD basic control.

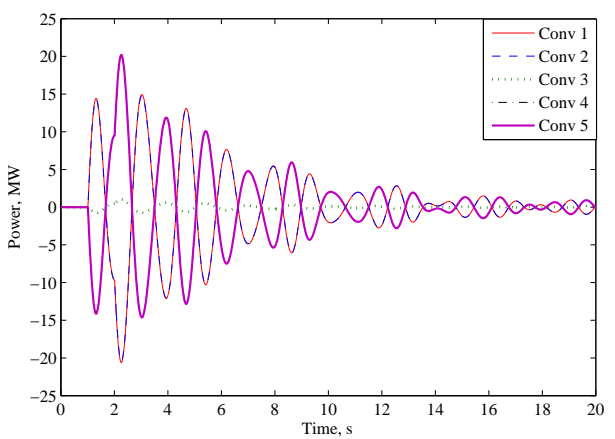

Figure 7. Power modulation of the terminals for the case of POD control using dc voltage loop shaping design.

\section{B. Case study 2}

A solid three phase to ground fault occurs at Bus 4031 occurs at $\mathrm{t}=1 \mathrm{~s}$ which is cleared at $\mathrm{t}=1.1 \mathrm{~s}$. The result of this disturbance is shown in Fig. 8. Clearly the new POD controller, based on closed loop dc voltage shaping, performs better than basic POD control as it utilizes the control direction of higher gain.

Clearly, the new dc voltage loop shaping design method has the advantage that it eliminates the communication between the terminals and drawbacks related to this.

It was identified that the new dc voltage loop shaping control method has similar performance as a centralized POD 


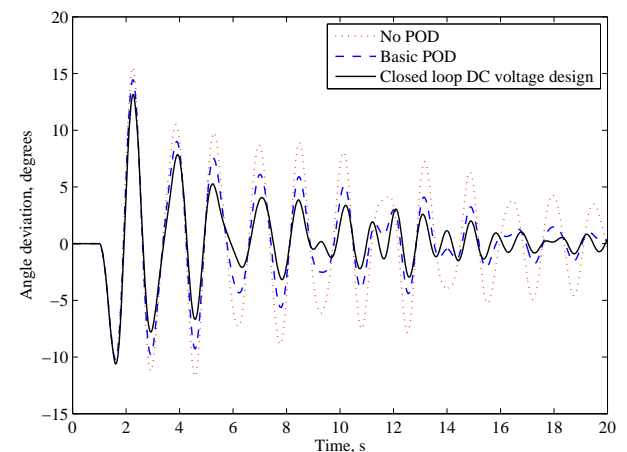

Figure 8. Angle difference of generators 4072 and 4063.

controller, under ideal case e.g. no communication delay, using communication. However, communication delay and data package loss may cause problems and even cause instability. In addition, it is hard to compensate for data latency as it is not always constant which emphasizes the great advantage with the proposed control structure.

\section{CONCLUSIONS}

This article presents a novel supplementary controller for electro-mechanical oscillation damping through active power modulation of MTDC systems.

The new method maximizes the relative controllability in which highest damping for each mode is achieved. The novel controller performs dc voltage loop shaping to maximize the relative controllability by only sending the control signal to one converter, which could be based on either local or widearea measurements. The new dc voltage loop shaping control method gets round the need of communication between the terminals and avoids the drawbacks, such as delay, related to this. Instead the other converters react upon a change in the dc voltage, in the direction of maximum relative controllability as a consequence to the novel closed loop shaping. Simulations performed in the Nordic32 test system implemented in PowerFactory/Digsilent including a five terminal MTDC system show promising results.

\section{REFERENCES}

[1] "Research and development plan - European Grid towards 2020 challenges and beyond," tech. rep., ENTSO-E, Brussels, March 2010. first edition.

[2] T. Haileselassie, K. Uhlen, and T. Undeland, "Control of multiterminal HVDC transmission for offshore wind energy," in Proc. Nordic Wind Power Conference Presentation, (Rønne, Denmark), Sept. 10-11, 2009.

[3] A. van der Meer, R. Hendriks, and W. Kling, "Combined stability and electro-magnetic transients simulation of offshore wind power connected through multi-terminal VSC-HVDC,' in Proc. IEEE PES GM '10, (Minneapolis, USA), pp. 1-7, July 25-29, 2010.

[4] J. Beerten and R. Belmans, "Modeling and control of multi-terminal VSC HVDC systems," Energy Procedia, vol. 24, pp. 123 - 130, 2012. Selected papers from Deep Sea Offshore Wind R\&D Conference, Trondheim, Norway, 19-20 January 2012.

[5] E. Prieto-Araujo, F. D. Bianchi, A. Junyent-Ferre, and O. GomisBellmunt, "Methodology for droop control dynamic analysis of multiterminal VSC-HVDC grids for offshore wind farms," IEEE Trans. Power Del., vol. 26, no. 4, pp. 2476-2485, 2011.

[6] R. Eriksson, V. Knazkins, and L. Söder, "On the assessment of the impact of a conventional HVDC on a test power system," in Bulk Power System Dynamics and Control - VII. Revitalizing Operational Reliability, 2007 iREP Symposium, pp. 1-5, 2007.
[7] S. Azad, R. Iravani, and J. Tate, "Damping inter-area oscillations based on a model predictive control (mpc) hvdc supplementary controller," Power Systems, IEEE Transactions on, vol. 28, no. 3, pp. 3174-3183, 2013.

[8] F. de Mello, P. J. Nolan, T. Laskowski, and J. Undrill, "Coordinated application of stabilizers in multimachine power systems," Power Apparatus and Systems, IEEE Transactions on, vol. PAS-99, no. 3, pp. 892901, 1980.

[9] P. Kundur, Power System Stability and Control. McGraw-Hill Inc, New York, 1993.

[10] K. Tomiyama, M. Sato, K. Yamaji, M. Sekita, and M. Goto, "Power swing damping control by HVDC power modulation in an AC/DC hybrid transmission system," Electrical Engineering in Japan, vol. 124, no. 3, pp. 10-18, 1998

[11] K. Uhlen, S. Elenius, I. Norheim, J. Jyrinsalo, J. Elovaara, and E. Lakervi, "Application of linear analysis for stability improvements in the nordic power transmission system," in Power Engineering Society General Meeting, 2003, IEEE, vol. 4, p. 4 vol. 2666, july 2003.

[12] R. Eriksson and L. Söder, "Coordinated control design of multiple HVDC links based on model identification," Computers and Mathematics with Applications, Elsevier, vol. 60, pp. 944-953, Aug. 2010.

[13] "The European electricity grid initiative (EEGI)," tech. rep., ENTSO-E, 2010.

[14] Working Group 14.29, "149 Coordination of controls of multiple FACTS/HVDC links in the same system," tech. rep., Cigré, 1999.

[15] R. Eriksson, Coordinated Control of HVDC Links in Transmission Systems. PhD thesis, KTH Royal Institute of Technology, Stockholm, Sweden, 2011.

[16] R. Eriksson, "On the centralized nonlinear control of HVDC systems using lyapunov theory," Power Delivery, IEEE Transactions on, vol. 28, no. 2, pp. 1156-1163, 2013.

[17] R. Eriksson, Coordinated control of HVDC links in transmission systems. $\mathrm{PhD}$ thesis, KTH Royal Institute of Technology, Mar. 2011.

[18] J. Machowski, P. Kacejko, L. Nogal, and M. Wancerz, "Power system stability enhancement by wams-based supplementary control of multi-terminal hvdc networks," Control Engineering Practice, vol. 21, pp. 583-592, 2013.

[19] R. Eriksson, J. Beerten, M. Ghandhari, and R. Belmans, "Optimising dc voltage droop settings for ac/dc system interactions," IEEE Transactions on Power Delivery, 2013.

[20] R. E. Best, Phase-Locked Loops: Design, Simulation and Applications. McGraw-Hill Inc, 2007.

[21] R. Pinto, P. Bauer, S. Rodrigues, E. Wiggelinkhuizen, J. Pierik, and B. Ferreira, "A novel distributed direct-voltage control strategy for grid integration of offshore wind energy systems through mtdc network," Industrial Electronics, IEEE Transactions on, vol. 60, no. 6, pp. 24292441, 2013.

[22] F. Pagola, I. Perez-Arriaga, and G. C. Verghese, "On sensitivities, residues and participations: applications to oscillatory stability analysis and control," Power Systems, IEEE Transactions on, vol. 4, pp. 278-285, Feb 1989

[23] J. L. Domínguez-García, C. E. Ugalde-Loo, F. Bianchi, and O. GomisBellmunt, "Input-output signal selection for damping of power system oscillations using wind power plants," International Journal of Electrical Power \& Energy Systems, vol. 58, no. 0, pp. 75 - 84, 2014.

[24] J. Dominguez-Garcia, F. Bianchi, and O. Gomis-Bellmunt, "Control signal selection for damping oscillations with wind power plants based on fundamental limitations," Power Systems, IEEE Transactions on, vol. 28, pp. 4274-4281, Nov 2013.

[25] R. Eriksson and L. Söder, "Wide-area measurement system-based subspace identification for obtaining linear models to centrally coordinate controllable devices," Power Delivery, IEEE Transactions on, vol. 26, pp. 988-997, Apr. 2011.

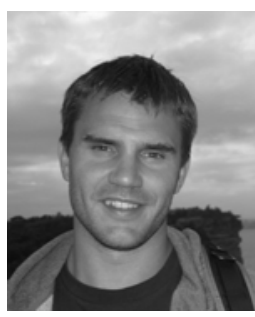

Robert Eriksson received the M.Sc. and Ph. D. degrees in Electrical Engineering from the KTH Royal Institute of Technology, Stockholm, Sweden, in 2005 and 2011 respectively.

$\mathrm{He}$ is currently holding a position as Associate Professor in the Center for Electric Power and Energy (CEE), DTU Technical University of Denmark. His research interests include power system dynamics and stability, HVDC systems, dc grids and automatic control. 